\title{
Análise Crítica e Sistemática sobre Técnicas Computacionais para a Detecção Automática de Pontos Cefalométricos
}

\author{
$\underline{\text { João Victor Oliveira Couto }}^{1}$ e Michele Fúlvia Angelo ${ }^{2}$ \\ 1. Bolsista FAPESB, Graduando em Engenharia de Computação, Universidade Estadual de Feira de Santana, e-mail: \\ jictyvoo.ecomp@gmail.com \\ 2. Orientador, Departamento de Ciências Exatas, Universidade Estadual de Feira de Santana, e-mail: \\ mfangelo@ecomp.uefs.br
}

PALAVRAS-CHAVE: cefalometria; pontos cefalométricos; técnicas computacionais.

\section{INTRODUÇÃO}

A análise cefalométrica, baseada em radiografias laterais da face, é uma ciência que faz parte do ramo odontológico, que visa obter dados referentes a localização e posição de pontos cefalométricos, para que a partir dos mesmos seja realizado um diagnóstico pelos profissionais da área [CHUKRUBURTTY S. et al].

De acordo com Ren et al (1998), existem cerca de 70 pontos de marcação em uma imagem cefalométrica comum, e esse grande número de pontos demanda uma grande quantidade de tempo para que os profissionais realizem sua identificação e marcação.

O objetivo deste trabalho é realizar um estudo crítico e sistemático de técnicas de detecção automática de pontos cefalométricos a fim de se identificar as que apresentam melhores resultados para um maior número de pontos cefalométricos.

\section{METODOLOGIA}

O levantamento bibliográfico necessário para a realização deste trabalho foi realizado utilizando duas ferramentas de pesquisa: o Google Schoolar e o Periódicos Capes. A pesquisa foi constituída pelas seguintes palavras chave: automatic cephalometric measurement; cephalometric points; medição automática de pontos cefalométricos; e pontos cefalométricos.

A partir dos resultados obtidos utilizando as palavras-chave apresentadas, foi realizada uma seleção dos artigos a serem analisados na pesquisa. A seleção inicial dos artigos foi reunida a partir da leitura de seus abstracts.

A busca pela bibliografia necessária resultou em uma base contendo 42 artigos publicados entre os anos de 1998 e 2017. Esses artigos selecionados, inicialmente, deveriam atender ao critério exigido quanto ao idioma, o qual refere-se à publicações escritas no idioma inglês e/ou português. Dessa forma, obtiveram-se 2 artigos publicados na língua portuguesa e 40 artigos publicados na língua inglesa.

Visando facilitar a análise de todos os artigos lidos, foi criada uma planilha e um arquivo de texto contendo os dados mais importantes referentes à cada artigo lido, ordenando-os por data de publicação e ordem alfabética. Os dados salvos na planilha foram: Ano de Publicação; Nome do Trabalho; Técnicas utilizadas; Número de Radiografias utilizadas; Número de Pontos Cefalométricos encontrados; e Todos os pontos encontrados pelo algoritmo. 


\section{RESULTADOS E DISCUSSÃO}

A análise dos artigos foi realizada de forma linear ao tempo de publicação dos mesmos, com o intuito de analisar o avanço das técnicas e tendências com o passar dos anos. Para uma melhor visualização dos dados referentes à cada publicação, as mesmas foram divididas por abordagens distintas, as quais configuram-se como: 1 - Abordagens Baseadas em Modelos; 2 - Abordagens Baseadas em Computação Flexível; 3 Abordagens Híbridas.

O resultado dessa divisão dos artigos, bem como os dados obtidos (quantidade de radiografias utilizadas e pontos encontrados), podem ser visualizados na Tabela 1.

Tabela 1 - Artigos por ano, métrica, quantidade de radiografias e pontos encontrados

\begin{tabular}{cccc}
\hline Ano Artigo & Quantidade de & Quantidade de & Métrica \\
& Radiografias & Pontos encontrados & \\
& &
\end{tabular}

\begin{tabular}{|c|c|c|c|c|}
\hline \multicolumn{5}{|c|}{ Abordagens Baseadas em Modelos } \\
\hline 1998 & RUDOLPH, D.J. et al & 14 & 15 & $\begin{array}{c}\text { Pixel, } \\
\text { Milímetros }\end{array}$ \\
\hline 2000 & HUTTON, TIM J. et al & 63 & 16 & Milímetros \\
\hline 2003 & CHUKRUBURTTY, S. et al & 130 & 8 & Porcentagem \\
\hline 2006 & KAZANDJIANA, S. et al & 20 & 10 & Milímetros \\
\hline \multicolumn{5}{|c|}{ MARIANO } \\
\hline 2008 & GALHOOD M. et al & 200 & 15 & Porcentagem \\
\hline 2010 & TRPOVSKI, Ž. et al & 131 & 17 & Milímetros \\
\hline \multicolumn{5}{|c|}{ Abordagens Baseadas em Computação Flexível } \\
\hline 2008 & BABA, M. S. et al & 20 & 11 & Milímetros \\
\hline 2009 & LEONARDI, R. et al & 41 & 10 & Milímetros \\
\hline 2009 & TANIKAWAA, C. et al & 465 & 20 & Milímetros \\
\hline \multicolumn{5}{|c|}{ Abordagens Híbridas } \\
\hline 2013 & KAUR, A. et al & 85 & 18 & Milímetros \\
\hline 2014 & IBRAGIMOV, B. et al & 200 & 18 & Milímetros \\
\hline 2001 & $\begin{array}{c}\text { GRAU, V; ALCAÑIZ M, JUAN, } \\
\text { MC; MONSERRAT, C; KNOLL, } \\
\text { C }\end{array}$ & 20 & 17 & Milímetros \\
\hline 2005 & AHMED, A. S. et al & 27 & 18 & Milímetros \\
\hline
\end{tabular}

A quantidade de pontos encontrados varia conforme a abordagem utilizada, devido ao fato de algumas abordagens conseguirem encontrar pontos mais facilmente do que outras abordagens. Dessa forma, percebe-se a importância de abordagens híbridas, que tiveram sua utilização mais abordada nos últimos anos em comparação às outras 
abordagens apresentadas, bem como o fato de que os pontos mais fáceis de serem encontrados, são aqueles que por sua vez são os mais escolhidos para realizar a busca e medição durante o treinamento e validação de novas técnicas.

Após a realização das análises sobre os artigos selecionados, foi possível notar os pontos cefalométricos mais comumente buscados pelos sistemas de marcação automática dos pontos cefalométricos, os quais encontram-se na Tabela 2.

Tabela 2 - Pontos Cefalométricos comumente buscados por sistema de marcação automática

\begin{tabular}{|c|c|c|}
\hline Nome dos Pontos & Sigla & Descrição \\
\hline Ponto A & A & $\begin{array}{c}\text { Ponto da concavidade mais profunda da maxila, } \\
\text { encontra-se entre a espinha nasal anterior e a prótese }\end{array}$ \\
\hline Ponto B & B & $\begin{array}{c}\text { Ponto da concavidade mais profunda da sínfise } \\
\text { mandibular, encontra-se entre a infradentale e o pogonion }\end{array}$ \\
\hline Nasio & N & O ponto mais anterior da sutura fronto-nasal \\
\hline Pório & Po & ponto mais superior do meato acústico externo \\
\hline Pogônio & Pog & O ponto mais anterior do contorno anterior da sínfise \\
\hline mentoniano & Me & O ponto mais inferior da sínfise mandibular \\
\hline Sela & S & O ponto localizado no centro geométrico da sela túrcica \\
\hline Espinha Nasal & ENP & ponto localizado na porção mais posterior da maxila óssea \\
\hline Posterior & PL & O ponto mais proeminente do nariz \\
\hline Pró-nasal & Pn & O ponto onde a borda inferior do nariz encontra o contorno \\
\hline Subnasal & Sn & O ponto mais anterior do lábio superior \\
\hline Lábio Superior & UL & O ponto mais retraído do queixo ósseo \\
\hline Pogônio Mole & Pog inferior & Ontorior do lábio inferior \\
\hline
\end{tabular}

Devido ao grande número de pontos cefalométricos, e suas localizações distintas, as formas de medição para cada um dos mesmos também é distinta, de forma que cada técnica desenvolvida possui diferentes valores de erro médio para cada ponto medido, sendo esses valores mensurados como a diferença entre a medição manual realizada por um profissional da área, e a medição realizada pelo sistema proposto. Levando-se em consideração apenas os pontos cefalométricos mais buscados nos artigos, é possível afirmar que as abordagens híbridas possuem uma maior constância quanto ao erro médio calculado para cada ponto medido. Além disso, as abordagens híbridas revelaram possuir uma taxa de erro médio mais constante e menos mutável que as outras abordagens, de uma forma geral. Dessa forma, as técnicas híbridas abrem um espaço de pesquisa bem maior para o desenvolvimento de novos algoritmos para marcação automática de pontos.

\section{CONCLUSÃO}


Apesar da baixa flexibilidade para o desenvolvimento de novas técnicas computacionais para realizar a identificação automática dos pontos cefalométricos e tratamento das imagens, é notado que a junção de métodos já bem discutidos e fomentados na comunidade acadêmica da área, bem como diferentes formas de implementação dos mesmos, trazem melhorias e maiores acertos na identificação dos pontos. Isso se deve ao fato de algumas técnicas suprirem o ponto fraco de outras, de forma a tornar-se mais completa quando unificada à outra técnica.

\section{REFERÊNCIAS}

CHUKRUBURTTY S., YAGI M., SHIBATA T., CAUWENBERGHS G.Robust Cephalometric Landmark Identification Using Support Vector Machines, 2003

EL-FEGH I., GALHOOD M., SID-AHMED M., AHMADI M. Automated 2-D Cephalometric Analysis of X-ray by Image Registration Approach based on Least Square Approximator, 30th Annual International IEEE EMBS Conference, 2008

GRAU V, ALCAÑIZ M, JUAN MC, MONSERRAT C, KNOLL C. Automatic localization of cephalometric landmarks. J Biomed Inform. 34(6):146-56, 2001

HUTTON T. J., CUNNINGHAM S., HAMMOND P. An evaluation of active shape models for the automatic identification of cephalometric landmarks, European Journal of Orthodontics 22 499-508, 2000

IBRAGIMOV B., LIKAR B., PERNUŠ F., VRTOVEC T. Automatic Cephalometric X-Ray Landmark Detection by Applying Game Theory and Random Forests, Springer-Verlag Berlin Heidelberg, 2014

KAUR A., SINGH C. Automatic cephalometric landmark detection using Zernike moments and template matching, Springer-Verlag London, 2013

KAZANDJIANA S., KILIARIDISB S., MAVROPOULOSA A. Validity and Reliability of a New Edge-based Computerized Method for Identification of Cephalometric Landmarks, Angle Orthodontist, Vol. 76, No. 4, 2006

LEONARDI R., GIORDANO D., MAIORANA F. An Evaluation of Cellular Neural Networks for the Automatic Identification of Cephalometric Landmarks on Digital Images, 2009

MOSLEH M. A. A., BABA M. S., HIMAZIAN N., AL-MAKRAMANI B. M. A. An Image Processing System for Cephalometric Analysis and Measurements, 2008

REN, J., LID D., FENG D. , SHAO J., ZHAO R., LIAO YI, LIN Z. A Knowledge-based Automatic Cephalometric Analysis Method, Proceedings of the 20th Annual International Conference of the IEEE Engineering in Medicine and Biology Society, Vol. 20, No 2, 1998

RUDOLPH D.J., SINCLAIR P.M., COGGINS J.M. Automatic computerized radiographic identification of cephalometric landmarks, American Journal of Orthodontics and Dentofacial Orthopedics, Vol. 113, No. 2, 1998

RUEDA, SYLVIA \& ALCAÑIZ, MARIANO, An Approach for the Automatic Cephalometric Landmark Detection Using Mathematical Morphology and Active Appearance Models, MICCAI 2006, LNCS 4190, pp. 159-166, 2006

SAAD A. A., EL-BIALY A., KANDIL A. H., AHMED A. S. Automatic Cephalometric Analysis Using Active Appearance Model and Simulated Annealing, GVIP 05 Conference, 2005

TANIKAWA C., YAGI M., TAKADA K. Automated Cephalometry: System Performance Reliability Using Landmark-Dependent Criteria, Angle Orthodontist, Vol. 79, No. 6, 2009 
VUPINIØ P., TRPOVSKI Ž., ŠØEPAN I. Automatic landmarking of cephalograms using active appearance models, European Journal of Orthodontics, Vol. 32, p. 233-241, 2010 